\title{
Características clínicas de pacientes diabéticos manejados ambulatoriamente en el Instituto Salvadoreño del Seguro Social
}

\author{
DOI 10.5377/alerta.v5i1.10364 \\ Víctor David Franco ${ }^{1 *}$, Claudia López de Blanco ${ }^{2}$ \\ 1-2. Departamento de Investigación y Docencia en Salud, Instituto Salvadoreño del Seguro Social, San Salvador, El Salvador \\ ${ }^{*}$ Correspondencia \\ $\square$ victor.franco@isss.gob.sv \\ 1. (1) $0000-0003-4087-838 \mathrm{X}$
}

\begin{abstract}
Resumen
Introducción. En 2019 la Federación Internacional de Diabetes reportó 463 millones de diabéticos a escala mundial, y en Centroamérica 19252 000. Ese año se brindaron 170230 consultas por diabetes en el Instituto Salvadoreño del Seguro Social; entre 2016-2019 se invirtieron \$22,5 millones en sus medicamentos, sin embargo, existe poca información sobre su clasificación clínica y ubicación geográfica. Objetivo. Definir características clínico-epidemiológicas de pacientes diabéticos manejados de manera ambulatoria. Metodología. Estudio descriptivo transversal con información del Departamento de Actuariado y Estadística sobre hospitalizaciones y atención ambulatoria de pacientes diabéticos mayores de 18 años para clasificarlos y ubicarlos geográficamente entre enero a junio de 2020. Los resultados se presentan en tablas aplicando medidas de tendencia central. Resultados. Fueron 10376 pacientes, 58,1 \% mujeres, de las cuales 76,2 \% tenía diabetes tipo 2. La edad promedio de los pacientes con diabetes tipo 1 es de 61 años y 57 años para los pacientes con diabetes tipo 2. El $61,8 \%$ de los pacientes con diabetes tipo 1 y el 70,2 \% de los pacientes con diabetes tipo 2, no presentaron complicaciones. Entre los que sí reportaron predominaron las nefropatías (diabetes tipo 1) y complicaciones múltiples (diabetes tipo 2). Las principales comorbilidades fueron hipertensión (61,7\%), dislipidemias (31,8\%) y enfermedad tiroidea (11\%). Un 44,6 \% provino de San Salvador, 35,8 \% de Santa Ana y 8,2 \% de La Libertad. Conclusiones. Existe mayor cantidad de mujeres con diabetes; en edad adulta predomina la diabetes tipo 2 con daños múltiples. Un 2,1 \% no está clasificado, la mayoría provino de San Salvador, la población más afectada está en Santa Ana.
\end{abstract}

Palabras clave

Diabetes mellitus, epidemiologia, complicaciones

\begin{abstract}
Introduction. In 2019, the International Diabetes Federation reported 463 million diabetics worldwide, and in Central America 19252 000. That year, 170230 consultations for diabetes were provided at the Salvadoran Social Security Institute, between 2016-2019 \$22,5 were invested million on their medications, yet little information exists on their clinical classification and geographic location. Objective. Define clinical-epidemiological characteristics of diabetic patients managed on an outpatient basis. Methodology. Descriptive cross-sectional study with information from the Actuarial and Statistical Department on hospitalizations and outpatient care of diabetic patients over 18 years of age to classify and locate them geographically between January and June 2020. The results are presented in tables applying central tendency measures. Results. There were 10376 patients, 58,1 \% women, of which 76,2 \% had with type 2 diabetes, average age 61 for type 1 diabetics and 57 years for patients with type 2 diabetes. 61,8 \% of type 1 diabetics and 70,2\% of type 2 did not present complications. In those who did report, nephropathies (type 1 diabetes) and multiple complications (type 2 diabetes) predominated. The main comorbidities were hypertension (61,7\%), dyslipidemia (31,8 \%) and thyroid disease (11\%), 44,6 \% came from San Salvador, 35,8 \% from Santa Ana and 8,2 \% from La Libertad. Conclusions. There are more women with diabetes, in adulthood type 2 diabetes predominates with multiple damages. 2,1 \% are not classified, the majority came from San Salvador, the most affected population is in Santa Ana.
\end{abstract}

Keywords

Diabetes mellitus, epidemiology, complications

\section{Introducción}

La diabetes mellitus es una de las enfermedades crónicas endémicas no transmisibles que debido a su alta frecuencia se ha llegado a posicionar entre las principales enfermedades que afectan a cientos de millones de personas en todo el mundo, con incidencia, morbilidad y mortalidad en aumento'.
ACCESO ABIERTO

Clinical characteristics of diabetic patients with outpatient management at the Salvadoran Social Security Institute

Citación recomendada: Franco VD, López de Blanco C. Características clínicas de pacientes diabéticos manejados ambulatoriamente en el Instituto Salvadoreño del Seguro Social. Alerta. 2022;5(1): 33-42. DOI: 10.5377/alerta. v5i1.10364

Recibido:

1 de febrero de 2021

Aceptado:

17 de diciembre de 2021

Publicado:

27 de enero de 2022

Contribución de autoría: VDF': concepción, recolección de datos, diseño, análisis e interpretación de datos y revisión del artículo. $\mathrm{CLB}^{2}$ : revisión del artículo y aprobación de la versión final, asesoría metodológica y ética.

\section{Conflicto de intereses:}

Los autores declaran no tener conflicto de interés. 
En el 2019, la Federación Mundial de Diabetes (WDF por sus siglas en inglés) reportó que el 93 \% de la población mundial, cuyas edades oscilaban entre los 20 a 75 años, adolecían de algún tipo de diabetes mellitus, esta proporción equivale a más de 463 millones de personas, muchas en edad productiva. En Centroamérica, la diabetes mellitus afecta al 8,5\% de la población, es decir a más de 19252000 personas'.

La evolución natural de la diabetes comprende el desarrollo de lesiones crónicas que terminan en daños degenerativos que, ya se mantenga o no controlada, eventualmente lesiona considerablemente los tejidos de órganos blanco como ojos, riñón, corazón y nervios, y conllevan a complicaciones que necesitan ser estabilizadas en los hospitales o requieren de terapias sustitutivas como la diálisis, además produce compromisos vasculares y del sistema nervioso que pueden terminar en infecciones severas, amputaciones de miembros y trasplante de órganos, las cuales son causa de mayor morbilidad, suspensión laboral temporal o permanente.

La información sobre atenciones ambulatorias, disponible en el Departamento de Actuariado y Estadística del Instituto Salvadoreño del Seguro Social (ISSS), indica que en 2019 se brindaron 4871908 consultas y 170230 fueron por diabetes mellitus (3,5\%). El 60,7\% de las atenciones (103 429) se brindaron a mujeres. Debido a la magnitud y proporciones de la diabetes en esta población, se hace necesario realizar en el ISSS investigaciones para actualizar la situación de su condición y que al mismo tiempo permita saber el origen de estas personas. El objetivo principal del estudio consiste en definir las características epidemiológicas y clínicas de pacientes diabéticos manejados de manera ambulatoria.

\section{Metodología}

Este es un estudio descriptivo transversal sobre atenciones ambulatorias por diabetes mellitus entre los meses de enero a junio del 2020. La información proviene de la revisión de expedientes electrónicos de pacientes de consulta externa. Los centros seleccionados fueron los que cuentan con expediente electrónico como el consultorio de especialidades (donde está centralizada la atención de especialidades médicas y da cobertura nacional), Unidad Médica de Santa Ana, clínicas comunales de Ayutuxtepeque, Miramonte, Las Victorias, Mejicanos, San Antonio Abad y de la consulta externa del Hospital General.

Se revisaron los reportes facilitados por el Departamento de Actuariado y Estadística sobre la producción de consulta externa de estos centros. En ellos se menciona que 117 825 consultas fueron brindadas a 77724 pacientes por diversas causas, al analizar cada caso se descartaron 67348 personas que consultaban por otras enfermedades, lo que dejó 10376 pacientes que consultaron por diabetes mellitus, esto representa el 13,3\% de personas atendidas en estos centros y el $0,6 \%$ de la población adulta afiliada al ISSS Además, se complementó la información con registros del Departamento de Actuariado y Estadística sobre movimientos hospitalarios y registros de dispensación de medicamentos obtenidos de la Farmacia Central.

Las variables que se investigaron fueron: número de afiliación, edad, sexo, tipo de diabetes y enfermedades asociadas más frecuentes (hipertensión arterial, dislipidemias, trastorno metabólico de las purinas y de las pirimidinas, patologías tiroideas, cardiopatías, trastornos del ritmo cardiaco, cirrosis hepática e insuficiencia renal crónica). La presencia o no de estas variables fue dicotomizada como ausente (0) o presente (1), mientas que las variables cualitativas se registraron con su nombre y código CIE-10. La información fue recopilada en una hoja de cálculo de Excel, luego se procedió a definir la cantidad de pacientes que fueron atendidos por centro asistencial y las características clínicas y epidemiológicas. El análisis estadístico se realizó a través de promedios y desviación estándar con Microsoft Excel. El artículo cuenta con la autorización del Comité de Ética para la Investigación del Instituto Salvadoreño del Seguro Social (CEIS).

\section{Resultados}

En el periodo de estudio, en estos 8 centros se brindaron 117825 consultas a 77724 personas; de estas, 10376 fueron por diabetes, esto equivale al 13,3\% de la población adulta adscrita al ISSS y al 0,6\% de todos los beneficiarios cubiertos por el régimen del ISSS para el 2020 (Tabla1).

En la Tabla 2 y Figura 1, se observa que 6025 casos fueron mujeres (58,1\%), 7907 $(76,2 \%)$ presentaban diabetes tipo 2 y otros $222(2,13 \%)$ no fueron clasificados adecuadamente. La edad promedio fue de 61 años (mínima: 49, máxima: 75 años), y 8947 pacientes tenían entre 40 a 79 años.

Un total de 1388 pacientes (61,8\%) no presentaron complicaciones relacionadas con la diabetes tipo 1. De los pacientes con diabetes tipo 2, 5553 (70,2 \%) no presentaron complicaciones, y de los pacientes con diabetes no especificada, 167 (75,2 \%) tampoco presentaron complicaciones. En los casos en que sí se reportaron complicaciones, los trastornos glomerulares $(\mathrm{N} 08,3)$ y 
Tabla 1. Descripción de la población y centros de atención ambulatoria donde son manejados estos pacientes enero-junio, 2020.

\begin{tabular}{lccc}
\hline \multicolumn{1}{c}{ Centro } & Consultas n $(\%)$ & Pacientes & Diabéticos \\
\hline Consultorio de especialidades & $57653(48,9)$ & $40519(52,1)$ & $4760(45,9)$ \\
UM Santa Ana & $39315(33,4)$ & $23928(30,8)$ & $4278(41,2)$ \\
CC Ayutuxtepeque & $5499(4,7)$ & $3156(4,1)$ & $258(2,5)$ \\
CC Miramonte & $5382(4,6)$ & $3453(4,4)$ & $335(3,2)$ \\
CC Las Victorias & $3967(3,4)$ & $2481(3,2)$ & $242(2,3)$ \\
CC Mejicanos & $3690(3,1)$ & $2264(2,9)$ & $300(2,9)$ \\
CE Hospital general & $1544(1,3)$ & $1202(1,5)$ & $27(0,3)$ \\
CC San Antonio Abad & $775(0,7)$ & $721(0,9)$ & $176(1,7)$ \\
TOTAL & 117825 & 77724 & $10376(13,3)$ \\
\hline
\end{tabular}

UM = unidad médica, CC= clínica comunal, CE= consulta externa

Tabla 2. Características de la población con diabetes mellitus atendida en el consultorio de espacialidades, consulta externa del Hospital General, Unidad Médica Santa Ana y 5 clínicas comunales en estudio ( $n=10376$ ).

\begin{tabular}{|c|c|c|c|}
\hline \multirow{2}{*}{ Parámetro } & \multicolumn{3}{|c|}{ Frecuencia n (\%) } \\
\hline & General & Hombre & Mujer \\
\hline Pacientes con diabetes mellitus & 10376 & $4351(41,9)$ & $6025(58,1)$ \\
\hline Diabetes Tipo 1 & $2247(21,7)$ & $965(42,9)$ & $1282(57,1)$ \\
\hline Diabetes Tipo 2 & $7907(76,2)$ & $3292(41,6)$ & $4615(58,4)$ \\
\hline Diabetes mellitus sin clasificar & $222(2,1)$ & $94(42,3)$ & $128(57,7)$ \\
\hline \multicolumn{4}{|l|}{ Edad promedio } \\
\hline Diabetes Tipo 1 & $61,4(\mathrm{DE}: 13,6)$ & 61,7 (DE: 13,6) & 62,8 (DE: 12,9) \\
\hline Diabetes Tipo 2 & $57,3(14,1)$ & 59 (DE: 13,0) & 61,5 (DE: 13,2) \\
\hline Diabetes mellitus no especificada & $64,2(\mathrm{DE} 12,6)$ & 61,7 (DE: 13,6) & 63,7 (DE:11,8) \\
\hline \multicolumn{4}{|l|}{ Distribución por grupos de edad } \\
\hline$<15-39$ & $586(5,6)$ & $253(43,2)$ & $333(56,8)$ \\
\hline $40-59$ & $3757(36,2)$ & $1586(42,2)$ & $2171(57,8)$ \\
\hline $60-79$ & $5190(50)$ & $2149(41,4)$ & $3041(58,6)$ \\
\hline$>80$ & $843(8,1)$ & $363(43,1)$ & $480(56,9)$ \\
\hline \multicolumn{4}{|l|}{ Complicaciones registradas } \\
\hline Diabetes Tipo 1 & $2247(21,6)$ & $965(42,9)$ & $1282(57,1)$ \\
\hline Sin complicaciones & $1388(61,8)$ & $583(42,0)$ & $805(58,0)$ \\
\hline Complicaciones renales & $434(19,3)$ & $214(49,0)$ & $220(51,0)$ \\
\hline Complicaciones neurológicas & $305(13,5)$ & $113(37,0)$ & $192(63,0)$ \\
\hline Complicaciones múltiples & $57(2,53)$ & $25(44,0)$ & $32(56,0)$ \\
\hline \multirow{2}{*}{ Parámetro } & \multicolumn{3}{|c|}{ Frecuencia n (\%) } \\
\hline & General & Hombre & Mujer \\
\hline Complicaciones circulatorias periféricas & $27(1,2)$ & $16(59,2)$ & $11(40,8)$ \\
\hline Complicaciones oftálmicas & $13(0,58)$ & $9(69,2)$ & $4(30,8)$ \\
\hline Con complicaciones no especificadas & $9(0,4)$ & $3(33,0)$ & $6(67,0)$ \\
\hline Que complica el embarazo & $5(0,22)$ & 0 & $5(100)$ \\
\hline Con otras complicaciones específicas & $4(0,17)$ & $1(25,0)$ & $3(75,0)$ \\
\hline Asociada a desnutrición & $4(0,17)$ & $1(25,0)$ & $3(75,0)$ \\
\hline Con cetoacidosis & $1(0,04)$ & 0 & $1(100)$ \\
\hline Diabetes tipo 2 & 7907 (762) & $3292(41,6)$ & $4615(58,4)$ \\
\hline Sin complicaciones & $5553(70,2)$ & $2278(41,0)$ & $3275(59,0)$ \\
\hline Con complicaciones múltiples & $952(12,0)$ & $418(43,9)$ & $534(56,1)$ \\
\hline Con complicaciones neurológicas & $787(9,9)$ & $314(40,0)$ & $473(60,0)$ \\
\hline
\end{tabular}




\begin{tabular}{lccc} 
Con complicaciones renales & $228(2,90)$ & $122(53,5)$ & $106(46,5)$ \\
Con complicaciones no especificadas & $170(2,15)$ & $59(34,7)$ & $111(65,3)$ \\
Con complicaciones oftálmicas & $105(1,32)$ & $56(53,3)$ & $49(46,7)$ \\
Con otras complicaciones especificadas & $52(0,65)$ & $18(34,6)$ & $34(65,4)$ \\
Con complicaciones circulatorias periféricas & $26(0,33)$ & $14(53,8)$ & $12(46,2)$ \\
Asociada a desnutrición & $19(0,24)$ & $10(52,6)$ & $9(47,4)$ \\
Preexistente en el embarazo & $10(0,13)$ & 0 & $10(100)$ \\
Con coma & $3(0,04)$ & $2(66,7)$ & $1(33,3)$ \\
Con complicaciones renales y neurológicas & $1(0,01)$ & 0 & $1(100)$ \\
Con cetoacidosis & $1(0,01)$ & $1(100)$ & 0 \\
\hline Diabetes mellitus no especificada & $222(2,13)$ & $95(42,7)$ & $127(57,3)$ \\
Sin complicación & $167(75,2)$ & $63(37,7)$ & $104(62,3)$ \\
Con complicaciones renales & $25(11,3)$ & $15(60,0)$ & $10(40,0)$ \\
Con complicaciones neurológicas & $12(5,4)$ & $5(41,7)$ & $7(58,3)$ \\
Con complicaciones no especificadas & $11(4,95)$ & $9(81,8)$ & $2(18,2)$ \\
Con complicaciones múltiples & $5(2,25)$ & $3(60,0)$ & $2(40,0)$ \\
Con complicaciones circulatorias periféricas & $2(0,9)$ & 0 & $2(100)$ \\
\hline
\end{tabular}

Diabetes Tipo 1 (E10-109, E12, O240), Diabetes Tipo 2 (E11-119, E12, O241), Diabetes mellitus sin clasificar $(\mathrm{E} 142,144,145,147-149)$
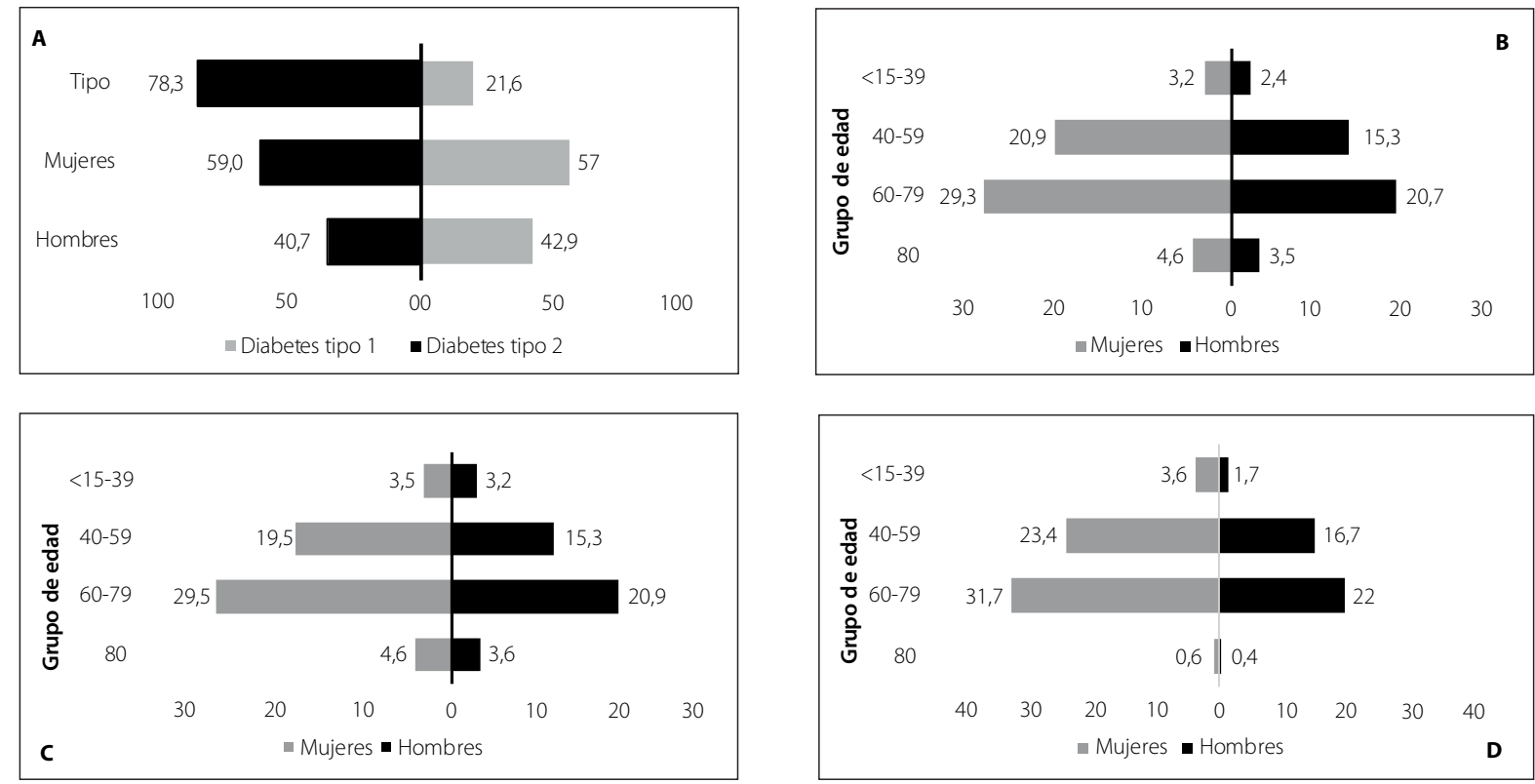

Figura1. Distribución de casos según (A) tipo de diabetes, (B) casos totales según sexo, (C) distribución de diabetes mellitus tipos I y II según grupo de edad y sexo (C y D).

nefritis túbulo intersticiales crónicas (N15,9) predominaron en pacientes con diabetes tipo 1 (424 casos equivalente al 19,3\%) y en 25 pacientes con diabetes no especificada $(11,3 \%)$. En los pacientes con diabetes tipo 2 predominaron las complicaciones múltiples (12\%). Las afecciones del sistema nervioso, como monopatías y polineuropatías diabéticas (CIE - 10: G59.0 y CIE - 10: G36.2), se detectaron en el $13,5 \%$ de los pacientes con diabetes tipo 1, en el 9,9\% de los pacientes con diabetes tipo 2 y en el 5,4\% de los pacientes con diabetes no especificada.
El análisis sobre enfermedades crónicas concomitantes indicó que estaban presentes en 12306 pacientes con diabetes. El número sobrepasa el total, puesto que algunos presentaban más de una condición concomitantemente con la diabetes, como hipertensión arterial (61,7\%), trastornos del metabolismo de las lipoproteínas y otras dislipidemias (31,8\%), enfermedad de la tiroides, hipotiroidismo e hipertiroidismo (11 $\%)$, purinas y pirimidinas (8,6 \%) y trastornos del ritmo cardiaco (1,4\%). Para complementar esta información se realizó un cruce de datos con otras fuentes de información asis- 
tencial, como la dispensación de medicamentos en Farmacia Central (Tabla 3).

Un 61,7\% (6403), además de recibir medicamentos para el control de la diabetes, recibió también para hipertensión arterial, otro 31,8 \% (3299) por trastornos del metabolismo de las lipoproteínas y otras dislipidemias, a la cabeza hipercolesterolemia y 1137 pacientes con enfermedades de la glándula tiroides, que en la mayoría se trataba de hipotiroidismo.

Otras condiciones presentadas en menor frecuencia por las que se les dispensaban medicamentos fueron las alteraciones en el metabolismo de las purinas y pirimidinas (888 casos), enfermedad cardiaca crónica (insuficiencia cardiaca, cardiopatía isquémica, cardiomiopatías diversas) y trastornos del ritmo (como flutter auricular, bloqueos de rama y taquicardias paroxísticas) en un $1,3 \%$ y 1,4\% respectivamente. También fueron detectadas otras condiciones como cirrosis hepática alcohólico-nutricional en un 0,27 \% e insuficiencia renal crónica en el $0,14 \%$, la cual no fue considerada como una complicación, sino como comorbilidad debido a la evolución clínica de los pacientes.

La mayoría de pacientes reside o es originaria principalmente de tres departamentos: San Salvador 4632 (44,6\%), con una tasa de 366 por 100000 habitantes; Santa Ana,
3999 (38,5 \%) y una tasa de 1021 por 100 000 habitantes; La Libertad, 851 (8,2 \%) con una tasa de 153 diabéticos / 100000 habitantes mayores de 18 años (Tabla 4).

\section{Discusión}

El estudio presenta los resultados de un análisis único que define por primera vez la situación epidemiológica y condición clínica de la diabetes mellitus en pacientes afiliados al ISSS y que son manejados ambulatoriamente.

El grupo de pacientes que fue atendido en los 8 centros de salud estudiados presentó características condicionantes de la enfermedad similares a las reportadas a escala mundial, como sexo, edad y otras enfermedades concomitantes. En lo referente a la predominancia de edad promedio y sexo, con algunas diferencias en frecuencia, las complicaciones y comorbilidades adolecidas son las mismas, pero hay algunas que deben ser sometidas a mayor escrutinio en los centros de atención ambulatoria, como la obesidad y sobrepeso. La mayoría de estos casos son vistos en los departamentos de San Salvador y Santa Ana, siendo en este último en donde parece estar concentrada la mayor cantidad de pacientes con diabetes tipo 1.

Tabla 3. Comorbilidades concomitantes registradas en la población de pacientes con diabetes mellitus en estudio.

\begin{tabular}{|c|c|c|}
\hline Comorbilidad & Frecuencia & Proporción \\
\hline Hipertensión arterial con/sin lesiones a órgano blanco & 6403 & $61,7 \%$ \\
\hline Trastornos del metabolismo de las lipoproteínas y otras lipidemias & 3299 & $31,8 \%$ \\
\hline Enfermedad tiroidea, hipo e hipertiroidismo, bocio, etc. & 1137 & $11,0 \%$ \\
\hline Trastornos del metabolismo de las purinas y de las pirimidinas & 888 & $8,6 \%$ \\
\hline $\begin{array}{l}\text { Trastornos ritmo cardiaco (flutter, bloqueo completo de rama, taquicardia su- } \\
\text { pra ventricular) }\end{array}$ & 145 & $1,4 \%$ \\
\hline $\begin{array}{l}\text { Enfermedad isquémica cardiaca, cardiopatía dilatada e insuficiencia cardiaca } \\
\text { congestiva }\end{array}$ & 133 & $1,3 \%$ \\
\hline Cirrosis hepática & 28 & $0,27 \%$ \\
\hline Insuficiencia renal crónica & 15 & $0,14 \%$ \\
\hline Osteoporosis & 10 & $0,10 \%$ \\
\hline Anemia por deficiencia de hierro & 9 & $0,09 \%$ \\
\hline $\begin{array}{l}\text { Enfermedades pulmonares, asma bronquial, enfermedad pulmonar obstruc- } \\
\text { tiva crónica }\end{array}$ & 9 & $0,09 \%$ \\
\hline Secuelas de accidente cerebro vascular & 8 & $0,08 \%$ \\
\hline Artritis reumatoide, no especificada & 3 & $0,03 \%$ \\
\hline Valvulopatías cardiacas (aortica, mitral) & 3 & $0,03 \%$ \\
\hline Obesidad & 3 & $0,03 \%$ \\
\hline Otros & 213 & $2,1 \%$ \\
\hline TOTAL & 12306 & \\
\hline
\end{tabular}


Tabla 4. Origen de los pacientes que son atendidos en los consultorios estudiados.

\begin{tabular}{lcccccc}
\hline Departamento & DM 1 & DM 2 & $\begin{array}{c}\text { DM no } \\
\text { clasificado }\end{array}$ & Total & Proporción & $\begin{array}{c}\text { Tasa / 100 000 habitantes } \\
\text { mayores de 18 años }\end{array}$ \\
\hline San Salvador & 796 & 3702 & 134 & 4632 & $44,64 \%$ & 366 \\
Santa Ana & 1037 & 2937 & 25 & 3999 & $38,54 \%$ & 1021 \\
La Libertad & 181 & 636 & 34 & 851 & $8,20 \%$ & 153 \\
Ahuachapán & 59 & 159 & 3 & 221 & $2,13 \%$ & 86 \\
La Paz & 53 & 87 & 4 & 144 & $1,39 \%$ & 55,4 \\
Sonsonate & 32 & 103 & 5 & 140 & $1,35 \%$ & 38,4 \\
Cuscatlán & 27 & 88 & 4 & 119 & $1,15 \%$ & 62,7 \\
San Vicente & 26 & 66 & 3 & 95 & $0,92 \%$ & 74,6 \\
Chalatenango & 17 & 40 & 2 & 59 & $0,57 \%$ & 45,8 \\
Usulután & 6 & 32 & 3 & 41 & $0,40 \%$ & 16,6 \\
Cabañas & 10 & 26 & 2 & 38 & $0,37 \%$ & 37 \\
San Miguel & 2 & 24 & 3 & 29 & $0,28 \%$ & 8,7 \\
La Unión & 0 & 5 & 0 & 5 & $0,05 \%$ & 3,2 \\
Morazán & 1 & 2 & 0 & 3 & $0,03 \%$ & 2,2 \\
TOTAL & 2247 & 7907 & 222 & 10376 & $100 \%$ & \\
\hline
\end{tabular}

La edad promedio de la población fue de 61 años o mayor, lo que concuerda con lo que se reporta en análisis similares verificados en poblaciones de Cuba, Costa Rica, Argentina y Brasil entre los años 2014 y $2016^{5,12,14,16,18,32}$ que ubicaron a la mayor parte de sus pacientes diabéticos adultos en esos rangos de edad. La distribución según el sexo de los pacientes indicó que la diabetes mellitus es una enfermedad con mayor número de pacientes mujeres, lo que coincide con muchos de los estudios consultados ${ }^{5}$. $6,9,10,13-16,18,20,33,34$ en donde más del $51 \%$ de casos son presentados en mujeres. No existe evidencia con suficiente robustez que demuestre que la condición de sexo es predisponente a adolecer diabetes. Lo anterior pudiera ser debido, entre otras cosas, a cambios en el estilo de vida de las mujeres, nutrición y sedentarismo generados por algunos tipos de trabajos (maquilas, fábricas, centros de llamadas, etc. $)^{4}$, a los que acceden cada vez más mujeres y esa condición ha generado un mayor número de personas de ese sexo en la cobertura del ISSS y por consiguiente se están detectando en mayor cantidad.

Según la condición de la enfermedad presente en los pacientes estudiados, la mayor frecuencia de casos son de diabetes tipo 2 y en menor cantidad del tipo 1. Esto guarda similitud con el predominio de la diabetes tipo 2 sobre la tipo 1 informado por la Federación Internacional de Diabetes en 2019', y también con otros análisis en poblaciones de diabéticos mexicanas ${ }^{15}$, peruanas ${ }^{2}$, brasileñas ${ }^{33}$ y ecuatorianas ${ }^{34}$ publicados entre el 2013 al 2019, en donde el predominio de la diabetes tipo 2 fue un hallazgo que quedó bien demostrado.
Por otro lado, la casuística de casos de diabetes tipo 1 aparentemente resultó ser una de las más altas registradas en América, superando a lo que reporta el atlas de la Federación Mundial de Diabetes y otras poblaciones estudiadas $1,8,15,26,29$, que no superan el $20 \%$. Solo en un estudio en diabéticos en el Ecuador ${ }^{34}$, llevado a cabo entre el 2014 y 2015 se detectó una proporción de diabéticos tipo 1 considerablemente mayor a la de la población de pacientes del ISSS. Lo anterior puede deberse a condiciones como el tamaño de la población que se estudió y a la manera que lo reporta el personal asistencial, de cualquier forma, este factor dificulta comparar nuestros hallazgos con otros estudios y con la población en general.

La frecuencia en que las afecciones del sistema nervioso fueron diagnosticadas en los pacientes es comparativamente menor a la de otros países que van desde un $8,5 \%$ (en pacientes diabéticos en Cuba en el $2018^{14}$ ) hasta un 56,6\% (detectado en estudios en el Perú en el $2016^{28}$ ). Solo un estudio en 2015 en poblaciones de diabéticos en el Ecuador reportó una proporción menor de casos del $0,38 \%{ }^{34}$.

El porcentaje de complicaciones renales difiere en 1,3\% en comparación a lo reportado en México en el $2013^{15}$, pues esta casuística es mayor al $19 \%$, y en este mismo sentido las nefropatías resultaron ser presentadas en mayor cantidad que el 3,9\% que reportaron los estudios de Perú ${ }^{3}$ y también en algunas poblaciones en España en el año 2017 del $6,3 \%{ }^{5}$ y Estados Unidos de 1,5\%?.

La frecuencia de retinopatía diabética reportada principalmente en la población con diabetes tipo 2 fue menor a otras se- 
ries $^{2-5,8,13-15,28,29}$ que hablan de proporciones entre el $25 \%$ y 48 \%. Una razón probable es que estos casos no están siendo adecuadamente registrados. Las afecciones vasculares periféricas resultaron ser inferiores que otras diagnosticadas en poblaciones del Perú, México Cuba y Ecuador que van desde un $1,2 \%$ en Ecuador hasta 10,8 \% en México ${ }^{3}$ $4,7,14,15,34$

Respecto a otras enfermedades presentadas concomitantemente, la hipertensión arterial figura como la más común, muy parecido a lo reportado mundialmente y superior al 23,3\% registrado en poblaciones en España ${ }^{9}, 27,3 \%$ en Perú en el $2007^{10}$, del $55,5 \%$ en Cuba ${ }^{6}$; y entre 54,5\% al 61,3\% en diabetes tipo 2 en México en $2014^{15}$.

La frecuencia de alteraciones del metabolismo de lípidos (dislipidemias como hipercolesterolemia, hipertrigliceridemia, etc.) fue el segundo estado comorbido más frecuentemente presentado por estos pacientes con una mayor frecuencia a lo registrado en personas diabéticas de España, Cuba y México ${ }^{5,14,15}$ que van del 15,3\% al 30,2\%. Algunos factores que pudieran estar determinando esta condición serían los cambios en los estilos de vida, el tipo de alimentación y el sedentarismo por mencionar algunos. El hipotiroidismo fue el tercer hallazgo en frecuencia y resultó superior al 2,1\% reportado en Perú en el $2012^{3}$ y del 9,45 \% en Paraguay en $2016^{19}$. En el 2018 en Perú, Marrero ${ }^{17}$, se reportó un porcentaje de pacientes diabéticos con enfermedad tiroidea que supera nuestra casuística con 32,4\%, de los cuales el 23,3\% fueron mujeres.

Otra serie de comorbilidades que fueron encontradas en la población estudiada, pero en menor frecuencia, fueron condiciones cardíacas crónicas, como cardiopatía isquémica, trastornos del ritmo e insuficiencia cardíaca congestiva, las cuales fueron menores a lo que indican los estudios llevados a cabo entre el 2006 al 2016 en países como México, Cuba, Perú y Chile, cuyos porcentajes de pacientes con diversos tipos de cardiopatías y diabetes mellitus van desde un $10 \%$ hasta el 78,3\%4,14,15,28. Algunas causas que podrían explicar lo anterior es que la mayoría de los pacientes adolecen de diabetes tipo 2 y tenían una edad que ronda los 50 años, donde probablemente no han desarrollado o no se les ha detectado aún estas condiciones crónicas propias de personas mayores además no se incluyó la totalidad de la población del ISSS.

La condición de obesidad y sobrepeso registrado en el 0,03\% en los pacientes sugiere que estas condiciones no están siendo adecuadamente monitoreadas y registradas, pues siendo estas frecuentes en una buena parte de la población adulta que consulta y que a menudo requieren de hospitalización en los centros del ISSS para su control, su frecuencia en los diabéticos fue marcadamente menor a la de otros estudios en donde se han reportado proporciones entre el 3,7\% en España ${ }^{2}$ hasta $89 \%$ en Estados Unidos ${ }^{8}$. Las complicaciones vasculares neurológicas o accidentes cerebro vasculares y sus secuelas acompañaron los casos de diabetes en una casuística bastante modesta si la comparamos con la de estudios realizados en México, Cuba, Paraguay y Perú, donde reportaron una incidencia que osciló entre un 4,4\% a 63,0 \% 4,14,21,23-25, probablemente porque muchos pacientes fallecieron antes de realizar el estudio debido al evento cerebral. En el caso de las complicaciones vasculares periféricas es posible que ya sufrieron amputaciones por lo que ya no son registrados, y también porque no se incluyó la totalidad de la población del ISSS.

La mayoría de diabéticos son originarios de los departamentos donde se encuentran los centros de desarrollo del país. Se identificaron a San Salvador, Santa Ana y La Libertad como los departamentos de dónde es originaria la mayoría de las personas estudiadas, siendo la población de Santa Ana la que presento la mayor cantidad de personas afectadas.

El número de pacientes, la condición de solo contar con información de 8 centros asistenciales y los vacíos en la clasificación de los pacientes, son las limitantes más importantes encontradas, por lo que son necesarios más estudios en los que se incluyan las poblaciones de la zona oriental del país. Este registro debería complementarse con los datos provenientes de los diferentes sistemas prestadores de salud que existen en el país para definir un diagnóstico situacional completo de la diabetes mellitus, sus tipos, complicaciones y comorbilidades presentadas. Aquí radica la principal limitante de nuestro análisis.

\section{Conclusiones}

Las condiciones de la población diabética del estudio presentaron como características más relevantes, el predominio de la diabetes mellitus tipo 2, con mayor frecuencia en mujeres y en las edades entre 60 y 79 años, con una edad promedio de 57,3 años. Se tuvo una prevalencia de casos de diabetes sin complicaciones, aunque las complicaciones que se presentaron con mayor frecuencia fueron las múltiples, seguidas de las neurológicas y renales. Las comorbilidades más relevantes fueron la hipertensión arterial seguida de los trastornos del metabo- 
lismo de las lipoproteínas y la enfermedad tiroidea. Del total de casos incluidos en el estudio predominaron los atendidos en el consultorio de especialidades, sin embargo, la mayor tasa de pacientes diabéticos procedía del departamento de Santa Ana.

\section{Agradecimiento}

Departamento de Actuariado y Estadística del ISSS, Dra. Claudia Suárez, por su apoyo en la gestión de la información.

\section{Financiamiento}

Autores declaran no tener fuente de financiamiento.

\section{Referencias bibliográficas}

1. Atlas de la Diabetes de la FID 9th ed. Federación Internacional de Diabetes. Bruselas, Bélgica. 2019. Disponible en: https://www.Diabetesatlas.org

2. Goday A. Epidemiologia de la Diabetes y sus complicaciones no coronarias. Rev Esp Card. 2002;55(6):657-670. DOI: $\underline{\text { 10.1016/ }}$ S0300-8932(02)76674-8

3. Ramos W, López T, Revilla L, More L, Huamaní M, Pozo M. Resultados de la vigilancia epidemiológica de Diabetes mellitus en hospitales notificantes del Perú. Rev. Perú. med. exp. 2012;31 (1):0915. Disponible en: http://www.scielo.org. pe/scielo.php?script=sci arttext\&pid =S1726-46342014000100002.

4. Sabag Ruiz E, Álvarez Félix A, Celiz Zepeda S, Gómez Alcalá A. Complicaciones crónicas en la Diabetes mellitus. Prevalencia en una unidad de medicina familiar. Revista Médica del Instituto Mexicano del Seguro Social. 2006;44(5):415-42. Disponible en: https://www.redalyc.org/articulo. oa?id=457745535005

5. Hechavarría Naranjo S, Achiong Estupiñán F, Méndez Gómez H, Vega Rico O, et al. Caracterización clínica epidemiológica de la Diabetes mellitus en dos áreas de salud. Municipio Cárdenas. 2017. Rev. Med. Electrón. 2019;41(4):899-913. Disponible en: $\underline{\text { http://scielo.sld.cu/scielo. }}$ php?script=sci arttext\&pid=S168418242019000400899\&lng=es

6. Dot Pérez Licet del C, Díaz Calzada M, Pérez Labrador J, Torres Marín J, Díaz Valdés M. Características clínico, epidemiológicas de la Diabetes mellitus en el adulto mayor. Policlínico Universitario Luís A Turcios Lima. Rev Ciencias Médicas. 2011;15(2):157-169. Disponible en: $\underline{\text { http://scielo.sld.cu/scielo. }}$ php? script $=$ sci arttext\&pid $=$ S156131942011000200015\&lng=es

7. Leal Ruiz E, Rodríguez Méndez L, Fusté Bruzain M. Complicaciones crónicas en pacientes con diagnóstico reciente de Diabetes mellitus tipo 2. Medicentro Electrónica. 2019;23(2):136-139. Disponible en: http://scielo.sld.cu/scielo. php?script=sci arttext\&pid=S102930432019000200136\&lng=es.

8. National Diabetes Statistics Report. Centers for Disease Control and Prevention. Atlanta, Estados Unidos. 2020. Disponible en: https://www.cdc.gov/Diabetes/data/ statistics/statistics-report.html

9. Gil Montalbán E, Zorrilla Torras B, Ortiz Marrón H, Martínez Cortés M, Donoso Navarro E, Nogales Aguado P, et al. Prevalencia de Diabetes mellitus y factores de riesgo cardiovascular en la población adulta de la Comunidad de Madrid: estudio PREDIMERC. Gac Sanit. 2010;24(3):233240. Disponible en: https://scielo.isciii. es/scielo.php?script=sci arttext\&pid =S0213-91112010000300010

10. García F, Solís J, Calderón J, Luque E, Neyra L, Manrrique $\mathrm{H}$, et al. Prevalencia de Diabetes mellitus y factores de riesgo relacionados en una población urbana. Rev soc Perú med interna. 2013;26(4):90-4. Disponible en: http://portal.revistas.bvs.br/index.php?mfn =3081\&about=access\&lang=pt\#

11. Vargas Uricoechea H,Casas-Figueroa L. Epidemiología de la Diabetes mellitus en Sudamérica: la experiencia de Colombia. Clínica e Investigación en Arteriosclerosis. 2016;28(5):245-256. DOI: 10.1016/j. arteri.2015.12.002

12. Centro Nacional de Información de Ciencias Médicas. Diabetes. Estadísticas Mundiales. Cuba. Biblioteca Médica Nacional. 2019. 14 p. Disponible en: http://files.sld.cu/ bmn/files/2019/06/factografico-de-saludjunio-2019.pdf

13. Graue Hernández E, Rivera De La Parra D, Hernández Jiménez S, Aguilar Salinas C, Kershenobich Stalnikowitz, D, Jiménez Corona A. Prevalence and associated risk factors of diabetic retinopathy and macular o edema in patients recently diagnosed with type 2 Diabetes. BMJ open ophthalmology. 2020;5(1)e000304. DOI: 10.1136/bmjophth-2019-000304

14. Pérez Ramírez L. Caracterización clínico epidemiológico de la diabetes mellitus tipo 2 en pacientes hospitalizados. Mayarí, 2016. Revista Médica Electrónica. 2018;40(6):18011818. Disponible en: http://scielo.sld.cu/ scielo.php?script=sci arttext\&pid=S1684$18242018000601801 \& \operatorname{lng}=$ es\&tlng=es

15. Cordero Hernández A, Pinto Almazán R. Diabetes mellitus tipo 1 y 2. Estudio epidemiológico del primer año del 
servicio de Consulta Externa del Hospital Regional de Alta Especialidad de Ixtapaluca. Diabetes. 2014;7(1):10. Disponible en: medigraphic.com/cgi-bin/new/resumen. cgi?IDARTICULO=49577

16. Cubero Alpízar C, Rojas Valenciano L. Comportamiento de la Diabetes mellitus en Costa Rica. Horiz. sanitario. 2017;16(3):211220. DOI: $10.19136 /$ hs.a16n3.1871.

17. Marrero A, Medina L, Alpízar R, RamosG. Disfunción tiroidea en pacientes con Diabetes mellitus tipo 2. Revista Finlay. 2020;10(3):222-230. Disponible en: http:// scielo.sld.cu/scielo.php?script=sci arttext\& pid $=\$ 2221-24342020000300222$

18. Centeno Maxzud M, Gómez Rasjido L, Fregenal M, Arias Calafiore F, Córdoba Lanus M, D'urso M, et al. Prevalencia de disfunción tiroidea en pacientes con diabetes mellitus tipo 2. Medicina Buenos Aires.2016;76(6):1-4. Disponible en: http:// www.medicinabuenosaires.com/revistas/ vol76-16/n6/355-358-Med76-5-6509Maxzud.pdf

19. Malvetti Maffei M, Báez Cabral S, Santa Cruz F. Disfunción tiroidea en pacientes con diabetes mellitus tipo 2: ¿Una asociación frecuente? Rev. virtual Soc. Parag. Med. Int. 2016;3(1):33-41. DOI: $\underline{10.18004 /}$ rvspmi/2312-3893/2016.03(01)33-041.

20. Loor Villavicencio C, Plaza Sánchez M. Incidencia de la anemia ferropénica (hierro sérico) en pacientes diabéticos adultos que acuden al laboratorio de análisis clínico Sánchez ubicado en el cantón Naranjito. Tesis de grado. Ecuador. Universidad de Guayaquil. 2018.188 p. Disponible en: http://repositorio. ug.edu.ec/bitstream/redug/34630/1/ BCIEQ-T-0328\%20Loor\%20Villavicencio\%20 Carlos\%20Dar\%c3\%ado\%3b\%20 Plaza\%20S\%c3\%a1nchez\%20Marlon\%20 Fabi\%c3\%a1n.pdf

21. González Pascual M, Barea R. Prevalencia de los factores de riesgo vascular entre los casos con Diabetes mellitus tipo 2 y sin Diabetes hospitalizados de 2011 a 2013 por accidentes cerebrovasculares. Endocrinología, Diabetes y Nutrición. 2019;66(3): 150-156. DOI: 10.1016/j. endinu.2018.10.008

22. Akhtar N, Kamran S, Singh R, Malik R, Deleu $D$, Bourke P, et al. The Impact of Diabetes on Outcomes After Acute Ischemic Stroke: A Prospective Observational Study. Journal of stroke and cerebrovascular diseases: the official journal of National Stroke Association. 2019;28(3):619-626. DOI: 10.1016/j.jstrokecerebrovasdis.2018.11.003

23. Sánchez Pando Y, Sánchez Nuñez R, Lugo Bencomo Y. Mortalidad por accidentes cerebrovasculares en el Hospital General Docente Abel Santamaría Cuadrado de Pinar del Río. Revista de Ciencias Médicas de Pinar del Río. 2020;24(1):6777. Disponible en: http://scielo.sld.cu/ scielo.php?script=sci arttext\&pid=S1561$\underline{31942020000100067 \& \operatorname{lng}=e n \& t \operatorname{lng}=e s}$.

24. Ramirez Zumaeta E. Factores de riesgo asociados a accidentes cerebrovasculares Hospital Regional Virgen de Fátima,

Chachapoyas. Universidad Nacional Toribio Rodríguez de Mendoza de Amazonas. 2019. Fecha de consulta: 2021. Disponible en: http://repositorio.untrm.edu.pe/handle/ UNTRM/1907

25. Real Delor R, Jara Castillo G. Pronóstico vital y secuelas neurológicas en los pacientes con accidente cerebrovascular del Hospital Nacional, Paraguay. Rev cubana med. 2016;55(3):181-189. Disponible en: http://scielo.sld.cu/scielo. php? script=sci arttext\&pid=S0034$75232016000300001 \& \operatorname{lng}=\mathrm{es}$.

26. Ruiz M, Escolar A, Mayoral E, Corral San Laureano F, Fernández-Fernández I. La Diabetes mellitus en España: mortalidad, prevalencia, incidencia, costes económicos y desigualdades. Gac. Sanit. 2006;20:15-24. DOI: $10.1157 / 13086022$

27. Cases Amenós A, Martínez Castelao A, Fort Ros J, Bonal Bastons J, Ruiz P, Vallés Prats $\mathrm{M}$ et al. Prevalencia de anemia y su manejo clínico en la enfermedad renal crónica estadios 3-5 no en diálisis en Cataluña: estudio MICENAS I. Nefrología (Madr.). 2014;34(2):189-198. Disponible en: http://scielo.isciii.es/scielo. php? script=sci arttext\&pid=S021169952014000200006\&lng=es.

28. Villena JE. Epidemiología de la diabetes mellitus en el Perú. Diagnostico. 2019;55(4):173-81. DOI: https://doi. org/10.33734/diagnostico.v55i4.21

29. Sapunar, J. Epidemiología de la Diabetes mellitus en Chile. Revista Médica Clínica Las Condes. 2016; 27(2):146-151. DOI: 10.1016/j. rmclc.2016.04.003

30. Arteaga A, Maiz A, Rigotti A, Cortés V. Asociación entre diabetes mellitus y patología cardiovascular en la población adulta de Chile: estudio de la Encuesta Nacional de Salud, 2009-2010. Rev. méd. Chile. 2014;142(2):175-183. DOI: $10.4067 /$ S0034-98872014000200005.

31. Cuevas A, Alonso R. Dislipidemia diabética. Rev. Med. Clin. Condes. 2016;27(2):152-159, DOI: 10.1016/j.rmclc.2016.04.004

32. Ferreira Gomes A, Vieira M, Rezende M, Oshima A, Muller M, Santos M et al. Perfil epidemiológico e nível de conhecimento de pacientes diabéticos sobre Diabetes e retinopatia diabética. Arq. Bras. Oftalmol. 2010;73(5):414-418. Disponible en: http://www.scielo.br/ 
scielo.php?script=sci arttext\&pid=S000427492010000500005\&lng=en

33. Macedo J, Oliveira A, Pereira I, Reis E, Assunção M. Epidemiological profile of Diabetes mellitus in northeastern Brazil. Research, Society and Development. 2019;8(3):e2883826. DOI: $10.33448 /$ rsdv8i3.826
34. Real Coto JJ, Ronceros Medrado SG, Gómez García MD, Roby Arias AJ, Idrovo Castro KJ, Ordoñez Zavala JM. Diabetes mellitus en pacientes atendidos en el hospital universitario: Caracterización epidemiológica. Revista científica INSPILIP. 2017;1 (2):1-18. DOI: 10.31790/inspilip. v1i2.21 